\title{
FINITE MONADIC ALGEBRAS
}

\author{
HYMAN BASS ${ }^{1}$
}

Introduction. The elementary structure theory for finite Boolean algebras is, for our purposes, summarized in the following theorem, (see $[1$, p. 163], or $[2$, p. 344]).

(*) If $B$ is a finite Boolean algebra, then it is completely characterized by the number of its elements, a number which must be of the form $2^{n}$, where $n$ is the number of atoms of $B$. In this case $B$ is faithfully represented as the Boolean algebra of all subsets of a set with $n$ elements. Moreover, $B$ is a free Boolean algebra if and only if $n$ is itself of the form $2^{r}$, in which case $B$ is free on $r$ generators.

In particular, a finitely generated Boolean algebra is finite. Recent work of Halmos, Tarski, and others in algebraic logic has engendered interest in the study of more general systems in which the Boolean structure is augmented by introducing certain additional operators. For instance, a closure algebra $A$ is a Boolean algebra with a unary operator $\exists: A \rightarrow A$ satisfying the axioms,

$$
\begin{aligned}
\exists 0 & =0, \\
p & \leqq \exists p, \\
\exists(p \vee q) & =\exists p \vee \exists q
\end{aligned}
$$

and

$$
\exists \exists p=\exists p
$$

for all $p$ and $q$ in $A$. Theorem 5.2 in [4] asserts that a free closure algebra on one free generator is infinite. We may show this directly by exhibiting any infinite closure algebra on one generator. To do so let $X$ be the real interval $[-1,1]$; we topologize $X$ by designating its family $C$ of closed sets.

$$
\begin{aligned}
& C=\{\phi,[0,0],[-1 / n, 1 / n],[-1 / n+1,1 / n],[-1 / n, 1 / n+1] \mid \\
& n=1,2, \cdots\} \text {. }
\end{aligned}
$$

Now we let $A$ be the Boolean algebra of all subsets of $X$ with $\exists p$ equal to the closure of $p$ in the topology defined by $C$. If $q=[0,1]$, ( $\exists q=[-1 / 2,1])$, then the closure subalgebra of $A$ generated by $q$ contains the entire infinite collection $C$.

Received by the editors May 1, 1957.

${ }^{1}$ National Science Foundation Fellow. 
A monadic algebra is a closure algebra with the additional axiom,

$$
\exists(\exists p)^{\prime}=(\exists p)^{\prime} .
$$

In a monadic algebra $\exists$ is called the quantifier, and its operation quantification. An elementary discussion in [3] shows that (0.1)-(0.5) are equivalent to the axioms: $(0.1),(0.2)$, and

$$
\exists(p \wedge \exists q)=\exists p \wedge \exists q .
$$

The finiteness question, answered above for Boolean and closure algebras, has remained unsettled for monadic algebras, which constitute a kind of intermediate case. A monadic algebra $A$ is said to be free on the set of generators $G \subset A$ if (i) $G$ generates $A$, and (ii) any map $f_{0}$ of $G$ into a monadic algebra $B$ can be extended to a monadic homomorphism $f: A \rightarrow B$. An immediate consequence of this definition is that $A$ is determined up to monadic isomorphism by the cardinality of $G$. Moreover, any monadic algebra with a generating set of cardinality at most that of $G$ would be a homomorphic image of such an A.

The principal result obtained here is a constructive description of all finitely generated free monadic algebras which, in particular, exhibits their finite cardinalities as a function of the number of free generators. This, of course, settles the finiteness question raised above. For completeness the construction includes a proof of freeness directly from the definition, and in the process a few mildly interesting results on monadic and Boolean homomorphisms are obtained which have reference to the representation theory developed by Halmos in [3]. ${ }^{2}$

We now proceed to define a few terms. If $A$ is a Boolean algebra and $p$ is in $A$, then $p$ is said to be an atom of $A$ if $p \neq 0$, and if every subelement of $p$ is either 0 or $p$. The following notation will also be convenient.

$$
p^{1}=p, \quad p^{-1}=p^{\prime}(=1-p) .
$$

If $\sigma=\left\{p_{1}, \cdots, p_{n}\right\}$ is a finite subset of $A$, we say that $\sigma$ is a partition of $p$ if (i) $p_{i} \wedge p_{j}=0$ for $i \neq j$, and (ii) $p_{1} \vee \cdots \vee p_{n}=p$. The nonzero elements of $\sigma$ are evidently distinct, and if $p=1$ they are just the atoms of the Boolean subalgebra of $A$ generated by $\sigma$.

1. Monadic forms and formal identities. It will now be convenient to introduce certain formal monadic expressions which will play a role analogous to that of polynomial forms in classical algebra. Let

2 The author is indebted to Professor Halmos for suggestion of the problem and for much valuable criticism. 
$\Delta=\{-1,1\}$, and let $E^{n}=\Delta \times \cdots \times \Delta$ ( $n$ times). For $e$ in $E^{n}$, we denote by $e_{i}$ the $i$ th coordinate of $e$, and for each $i$ we write $E_{i}^{n}$ $=\left\{e\right.$ in $\left.E^{n} \mid e_{i}=1\right\}, i=1, \cdots, n$. Regard now the symbols $x_{1}, \cdots, x_{n}$ as $n$ ordered monadic variables, let $X$ denote the vector $\left(x_{1}, \cdots, x_{n}\right)$, $\exists X$ the vector $\left(\exists x_{1}, \cdots, \exists x_{n}\right)$, and, for each such $X$, let $\langle X\rangle$ be the least class of finite sequences containing the one-term sequences $\left(x_{i}\right)$ and closed under the operations of prefixing $\exists$, infixing $\wedge$, and suffixing ${ }^{-1}$. We now define the maps,

$$
\lambda_{X}: E^{n} \rightarrow\langle X\rangle
$$

by $\lambda_{X}(e)=x_{1}^{e_{1}} \wedge \cdots \wedge x_{n}^{e_{n}}$, for all $e$ in $E^{n}$, and

$$
\mu_{X, i}: E_{i}^{n} \rightarrow\langle X\rangle
$$

by $\mu_{X, i}(e)=x_{i} \wedge \lambda \exists_{X}(e)$, for all $e$ in $E_{i}^{n}, i=1, \cdots, n$. We first observe:

(1.1) If $x_{1}, \cdots, x_{n}$ generate a Boolean algebra $B$, then $\left\{\lambda_{X}(e) \mid e\right.$ in $\left.E^{n}\right\}$ is a partition of 1 whose nonzero elements are just the atoms of $B$ (see, e.g., [2, p. 344]).

This apparatus is designed both for abbreviation of some unwieldy notation, and to facilitate the proofs of some useful formal results, to which we now proceed. If $A$ is a monadic algebra, then the range of $\exists, \exists(A)$, is a Boolean subalgebra of $A$ ([3, Theorem 5$])$ on which, by (0.4), $\exists$ induces the identity map. We have, therefore, for any $e$ in $E^{n}, \exists\left(\lambda_{\exists x}(e)\right)=\lambda \exists_{x}(e)$. Now using this together with (0.6), we obtain for each $e$ in $E_{i}^{n}, \exists\left(\mu_{X, i}(e)\right)=\exists x_{i} \wedge \lambda \exists x(e)$, and so, since $e_{i}=1$ for $e$ in $E_{i}^{n}$,

$$
\exists\left(\mu_{X, i}(e)\right)=\lambda_{\exists X}(e) .
$$

Suppose we consider now a Boolean algebra $B$ generated by the set $\left\{x_{i}, \bar{x}_{i} \mid i=1, \cdots, n\right\}$, under the assumptions, (i) $\left\{x_{1}, \cdots, x_{n}\right\}$ is a partition of 1 , and (ii) $\bar{x}_{i} \geqq x_{i}, i=1, \cdots, n$. By (1.1), the atoms of $B$ are all the nonzero elements of the form,

$$
\lambda_{X}(d) \wedge \lambda_{\bar{X}}(e)
$$

for $d$ and $e$ in $E^{n}$. By (i), (1.3) can be nonzero only if, for precisely one $i, d_{i}=1$, and, by (ii), it can be nonzero in this case only if also $e_{i}=1$ for the same $i$. By disjointedness, we may then simply delete the terms $x_{j}^{-1}$, for $j \neq i$, and write the resulting expression as $x_{i} \wedge \lambda_{\bar{X}}(e)$, where now $e$ must be in $E_{\imath}^{n}$ (and, of course, $\bar{X}=\left(\bar{x}_{1}, \cdots, \bar{x}_{n}\right)$ ). Now to summarize.

Lemma 1. If a Boolean algebra $B$ is generated by the set $\left\{x_{i}, \bar{x}_{i} \mid i\right.$ $=1, \cdots, n\}$ satisfying (i) and (ii) above, then $\left\{x_{i} \wedge \lambda_{\bar{X}}(e) \mid e\right.$ is in $E_{b}^{n}$, $i=1, \cdots, n\}$ is a partition of 1 containing the atoms "of $B$. 
Observe that if $B$ is a monadic algebra, and if $\bar{x}_{i}=\exists x_{i}$, then the resulting partition is just $\left\{\mu_{X, i}(e) \mid e\right.$ is in $\left.E_{i}^{n}, i=1, \cdots, n\right\}$. Moreover, in any finite Boolean algebra each element is (uniquely) the supremum of the atoms it contains. Make now the assumption that $\bar{x}_{i}=\exists x_{i}$.

CoRollary 1. $\lambda_{\exists X}(e)=V_{i} \mu_{X, i}(e)$, where the supremum is extended over all $i$ for which $e_{i}=1$. We write this $\lambda_{\exists X}(e)=\bigvee_{\left\{i \mid e_{i}=1\right\}} \mu_{X, i}(e)$.

Proof. If $e_{i}$ is 1 , then $\mu_{X, i}(e)=x_{i} \wedge \lambda \exists X(e) \leqq \lambda \exists X(e)$, and it can be easily checked that the other atoms of $B$ (i.e. $\mu_{X, j}(d)$ where $e \neq d \in E_{j}^{n}$ ) are disjoint from $\lambda_{\exists x}(e)$.

Corollary 2. $x_{i}=\mathrm{V}_{e \in E i^{n}} \mu_{X, i}(e)$.

Proof. Apply the same procedure as above.

For convenience we now choose, for each integer $r>0$, some definite enumeration $\left\{e^{k} \mid k=1, \cdots, 2^{r}\right\}$ of $E^{r}$, which shall henceforth remain fixed. If $A$ is a monadic algebra generated by elements $p_{1}, \cdots, p_{r}$, then we write $P=\left(p_{1}, \cdots, p_{r}\right)$, and $\lambda P=\left(\lambda_{P}\left(e^{1}\right), \cdots, \lambda_{P}\left(e^{n}\right)\right)$, where $n=2^{r}$, and we let $A_{0}$ be the Boolean subalgebra of $A$ generated by $\left\{\lambda_{P}\left(e^{k}\right), \exists \lambda_{P}\left(e^{k}\right) \mid k=1, \cdots, n\right\}$. Evidently $p_{1}, \cdots, p_{r}$ are in $A_{0}$. Moreover, $\left\{\lambda_{P}\left(e^{k}\right) \mid k=1, \cdots, n\right\}$ is a partition of 1 , so Lemma 1 applies and $\left\{\mu_{\lambda P, i}(e) \mid e\right.$ is in $\left.E_{i}^{n}, i=1, \cdots, n\right\}$ is a partition of 1 containing the atoms of $A_{0}$. By (1.2), $\exists\left(\mu_{\lambda P, i}(e)\right)=\lambda \exists_{\lambda P}(e) \in A_{0}$; i.e., $\exists$ maps the atoms of $A_{0}$ into $A_{0}$. Hence, by $(0.3), \exists\left(A_{0}\right) \subset A_{0}$, so $A_{0}$ is already a monadic subalgebra of $A$. Since $A_{0}$ contains a monadic generating set for $A$ we have $A_{0}=A$.

THEOREM 1. If $p_{1}, \cdots, p_{r}$ generate a monadic algebra $A$, then $\left\{\mu_{\lambda P, i}(e) \mid e\right.$ is in $\left.E_{i}^{n}, i=1, \cdots, n\right\}$ is a partition of 1 containing the atoms of $A$.

Corollary 3. A monadic algebra with $r$ generators has at most $n 2^{(n-1)}=2^{r} 2^{\left(2^{r}-1\right)}$ atoms (and hence at most $2^{\left[2^{r_{2}} 2^{\left(2^{r}-1\right)}\right]}$ elements).

Corollary 3 shows the significance of Theorem 1 (although the latter has other applications in the sequel); in particular, it settles the finiteness question referred to in the introduction.

2. Homomorphism theorems. Suppose $A$ and $B$ are Boolean algebras, $A$ finite, with $\sigma=\left\{p_{1}, \cdots, p_{n}\right\}$ and $\tau=\left\{t_{1}, \cdots, t_{n}\right\}$ partitions of 1 in $A$ and $B$, respectively. If $p_{i} \neq 0$, write $p_{i}=p_{i 1} \bigvee \cdots \vee p_{i m_{i}}$ as a supremum of atoms of $A$; if $p_{i}=0$, then define $m_{i}=1$. Consider now the map $f_{0}: \sigma \rightarrow \tau$ defined by $f_{0}\left(p_{i}\right)=t_{i}$.

Lemma 2. The map $f_{0}$ can be extended to a Boolean homomorphism 
$f: A \rightarrow B$ if and only if $t_{i}=0$ whenever $p_{i}=0$. In this case, there are at least $m_{1} \cdots m_{n}$ possible extensions $f$, and precisely this many when $B$ is finite and $\tau$ contains the atoms of $B$.

Proof. The necessity is an immediate consequence of the fact that $f(0)=0$. Suppose, conversely, that $t_{i}=0$ whenever $p_{i}=0$, and assume first that $\sigma$ contains all the atoms of $A$ : i.e. $m_{i}=1$ for all $i$. Then define $f: A \rightarrow B$ by $f\left(p_{i_{1}} \vee \cdots \vee p_{i_{k}}\right)=t_{i_{1}} \vee \cdots \vee t_{i_{k}}$. This $f$ is well defined since every element of $A$ is uniquely a supremum of atoms, and if extra $p_{i}$ 's which all equal zero are thrown in on the left, the corresponding $t_{i}$ 's, by hypothesis, contribute nothing new on the right. Moreover, $f$ clearly extends $f_{0}$ and commutes with all suprema. That $f$ commutes with complementation follows from the fact that the complement of a supremum of elements in a partition of 1 is just the supremum of the remaining elements in that partition. Therefore, $f$ is the desired homomorphism.

The general case reduces to this one by considering the new partitions $\sigma^{\prime}=\left\{p_{i j} \mid j=1, \cdots, m_{i} ; i=1, \cdots, n\right\}$ and $\tau^{\prime}=\left\{t_{i j} \mid j=1, \cdots\right.$, $\left.m_{i} ; i=1, \cdots, n\right\}$ where for each $i=1, \cdots, n, t_{i j_{i}}=t_{i}$ and $t_{i j}=0$ for $j \neq j_{i}, 1 \leqq j \leqq m_{i}$, and $j_{i}$ is an arbitrary integer between 1 and $m_{i}$ for each $i$. We are simply refining $\sigma$ to an atomic partition and compensation in $\tau$ by padding zeros in suitable places. The conditions for the first part of the proof are now fulfilled, and, for the $f$ so obtained, $f\left(p_{i}\right)=f\left(p_{i 1} \vee \cdots \vee p_{i n_{i}}\right)=t_{i 1} \vee \cdots \vee t_{i n_{i}}=t_{i}=f_{0}\left(p_{i}\right)$. The indicated freedom in the choice of the $j_{i}$ allows already $m_{i} \cdots m_{n}$ possible ways to extend $f_{0}$, and any two such clearly yield distinct $f$ 's. That these exhaust the possibilities when $B$ is finite and $\tau$ contains the atoms of $B$ follows from the fact that $f$ must preserve order and disjointedness. Namely, $f\left(p_{i j}\right)$ must be either 0 or $t_{i}$ since $f\left(p_{i j}\right) \leqq f\left(p_{i}\right)$ $=t_{i}$, an atom (excluding the trivial case $t_{i}=0$ ). By disjointedness, at most one $p_{i j}$ can map into $t_{i}$ and, moreover, at least one of them must if we are to have $f\left(p_{i}\right)$ equal to $t_{i}$ and not 0 ; this special element corresponds to the $p_{i j_{i}}$ above.

Corollary 4. A homomorphism of a finite Boolean algebra $A$ maps the atoms of $A$ one-to-one onto a partition of 1 , and conversely, any such map on the atoms of $A$ extends uniquely to a Boolean homomorphism.

We are now prepared to prove an extension theorem which is essential in the construction in III. Let $A$ and $B$ be monadic algebras generated by $\sigma=\left\{p_{1}, \cdots, p_{n}\right\}$ and $\tau=\left\{t_{1}, \cdots, t_{r}\right\}$, respectively. Consistently with the notation in Theorem 1 , we write $P$ $=\left(p_{1}, \cdots, p_{r}\right), T=\left(t_{1}, \cdots, t_{r}\right), \lambda P=\left(\lambda_{P}\left(e^{1}\right), \cdots, \lambda_{P}\left(e^{n}\right)\right)$, and $\lambda T=\left(\lambda_{T}\left(e^{1}\right), \cdots, \lambda_{T}\left(e^{n}\right)\right)$, where, recall, $n=2^{r}$. 
TheOREM 2. The map $f_{0}: \sigma \rightarrow \tau$ defined by $f_{0}\left(p_{i}\right)=t_{i}$ can be extended to a monadic homomorphism $f: A \rightarrow B$ if and only if $\mu_{\lambda T, i}(e)=0$ whenever $\mu_{\lambda P, i}(e)=0$, for all $e$ in $E_{i}^{n}, i=1, \cdots, n$.

Proof. Necessity is trivial since $\mu_{\lambda P, i}(e)$ and $\mu_{\lambda T, i}(e)$ have the same formal expression in terms of $\sigma$ and $\tau$, respectively, and we must have $f(0)=0$. Conversely, if we define,

$$
\begin{aligned}
f_{0}^{\prime}:\left\{\mu_{\lambda P, i}(e) \mid e \in E_{i}^{n}, i=1, \cdots, n\right\} \rightarrow\left\{\mu_{\lambda T, i}(e) \mid e \in E_{i}^{n},\right. \\
i=1, \cdots, n\}
\end{aligned}
$$

by $f_{0}^{\prime}\left(\mu_{\lambda P, i}(e)\right)=\mu_{\lambda T, i}(e)$, then, by Theorem 1 , Lemma 2 applies to $f_{0}^{\prime}$, which may therefore be extended to a Boolean homomorphism $f: A \rightarrow B$ by extending $f_{0}^{\prime}$ over suprema of atoms. To show that $f$ is monadic it suffices, by (0.3), to show that $f$ commutes with $\exists$ on atoms of $A$. By (1.2) and Corollary 1, each applied first in $A$ and then in $B$,

$$
\begin{aligned}
f\left(\exists \mu_{\lambda P, i}(e)\right) & =f\left(\lambda_{\exists^{\lambda P}}(e)\right)=f\left({\left.\underset{\left\{i \mid e_{i}=1\right\}}{\Lambda} \mu_{\lambda P, i}(e)\right)}=\underset{\left\{i \mid e_{i}=1\right\}}{\bigvee} \mu_{\lambda T, i}(e)=\lambda_{\exists \lambda T}(e)\right. \\
& =\exists \mu_{\lambda T, i}(e)=\exists f\left(\mu_{\lambda P, i}(e)\right) .
\end{aligned}
$$

It remains only to show that $f$ extends our original $f_{0}$. Now $p_{j}$ $=\mathrm{V}_{e^{k} \in E_{j}{ }^{r}} \lambda_{P}\left(e^{k}\right)$, and $t_{j}=\mathrm{V}_{e^{k} \in E_{j}{ }^{r}} \lambda_{T}\left(e^{k}\right)$. It therefore suffices to show that $f\left(\lambda_{P}\left(e^{k}\right)\right)=\lambda_{T}\left(e^{k}\right), k=1, \cdots, n$. By Corollary 2, where $X$ is replaced here by $\lambda P$ and $\lambda T$ successively, we have $\lambda_{P}\left(e^{k}\right)=\bigvee_{e \in E_{k}}{ }^{n} \mu_{\lambda P, k}(e)$ and $\lambda_{T}\left(e^{k}\right)=\mathrm{V}_{e \in E_{k}}{ }^{n} \mu_{\lambda T, k}(e)$, and hence,

$$
f\left(\lambda_{P}\left(e^{k}\right)\right)=\bigvee_{e \in E_{k}{ }^{n}} f\left(\mu_{\lambda P, k}(e)\right)=\bigvee_{e \in E_{k}{ }^{n}} \mu_{\lambda T, k}(e)=\lambda_{T}\left(e^{k}\right) .
$$

We digress here a moment to recall a result in [3] that will be used several times in the sequel. A Boolean subalgebra $B$ of a Boolean algebra $A$ is said to be relatively complete in $A$ if for every $p$ in $A$ the set $B(p)=\{q \mid q \in B, q \geqq p\}$ has an infimum, $\wedge B(p)$, in $B$. In particular, any finite, or, more generally, complete subalgebra $B$ is always relatively complete. Let $Q$ be the set of all quantifiers on $A$, and let $R$ be the set of all relatively complete subalgebras of $A$. For each $\exists$ in $Q$ define $f(\exists)=\exists(A)$, and for each $B$ in $R$ define $g(B): A \rightarrow A$ by $g(B) p=\Lambda B(p)$. The result we quote is the following:

(**) For $f$ and $g$ above, $f(Q)=R, g(R)=Q$, and $f=g^{-1}$. Thus, if $A$ is a monadic algebra and $p$ is in $A$, then

$$
\exists p=\wedge\{q \mid q \in \exists(A), p \leqq q\},
$$


and so $\exists$ is clearly order preserving. In particular, if $A$ is finite, then $\exists$ maps each atom of $A$ onto that unique atom of $\exists(A)$ above it. In general, when $\exists(A)$ is finite, $\exists$ maps an element onto the supremum of those atoms of $\exists(A)$ that the given element intersects nontrivially.

To resume, we shall now use Lemma 2 again to obtain a result bearing on Halmos' representation theory in [3]. If $A$ is a monadic algebra a constant, $c: A \rightarrow A$, is an idempotent endomorphism such that $c(A)=\exists(A)$. Then $c$ is said to be a witness for $p$ in $A$ if $c p=\exists p$, and we call $A$ rich if every $p$ in $A$ has a witness. Halmos proves his representation theorem ([3, Theorem 12$])$ directly only for rich monadic algebras, so it is of some interest to know whether certain classes of monadic algebras are rich. He gives reference to an example of a monadic algebra with no constants at all.

Let $A$ be a finite monadic algebra, and let $B=\exists(A)$. If $\tau$ $=\left\{t_{1}, \cdots, t_{n}\right\}$ is the atomic partition of 1 in $B$, let $\sigma=\left\{p_{i j} \mid j\right.$ $\left.=1, \cdots, m_{i} ; i=1, \cdots, n\right\}$ be the atomic partition of 1 in $A$, where $\sigma_{i}=\left\{p_{i 1}, \cdots, p_{i m_{i}}\right\}$ is the atomic partition of $t_{i}$ as an element of $A$. In this way we associate with $A$ the set $X(A)=\mathrm{X}_{i=1}^{n} \sigma_{i}$, a set with $N(A)=m_{1} \cdots m_{n}$ elements. $N(A)$ is clearly an algebraic invariant of $A$. Now if $x=\left(p_{1 j_{1}}, \cdots, p_{n j_{n}}\right)$ is any element of $X(A)$, we may associate with it the Boolean endomorphism, $c_{x}: A \rightarrow B(\subset A)$ which, by Corollary 4 , exists and is uniquely defined by

$$
c_{x}\left(p_{i j}\right)=\left\{\begin{array}{l}
0 \text { if } j \neq j_{i}, \\
t_{i} \text { if } j=j_{i},
\end{array} \quad j=1, \cdots, m_{1} ; i=1, \cdots, n .\right.
$$

Then $c_{x}\left(t_{i}\right)=c_{x}\left(p_{i 1}\right) \vee \cdots \vee c_{x}\left(p_{i m_{i}}\right)=t_{i}$, so by the uniqueness in Corollary $4, c_{x}$ induces the identity map in $B$, and hence also, $c_{x}(A)=B$; i.e., $c_{x}$ is a constant. Conversely, every constant $c$ determines an element $x_{c}$ of $X(A)$ such that $c_{x_{c}}=c$. To see this view $\tau$ as a partition of 1 in both $A$ and in $B$, and then apply the last part of the proof of Lemma 2 to show that $c\left(p_{i j}\right)=t_{i}$ for precisely one $p_{i j}$, $1 \leqq j \leqq n_{i}$, say $p_{i j_{i}}$. Then $x_{c}=\left(p_{1 j_{1}}, \cdots, p_{n j_{n}}\right)$ is clearly the desired element.

Theorem 3. A finite monadic algebra $A$ is rich. Moreover, there is a natural one-to-one correspondence between $X(A)$ and the $N(A)$ constants of $A$. Given any constant $c$ of $A$, there is an element $p$ in $A$ for which $c$ is the only witness.

Proof. It remains only to show that (i) every $p$ in $A$ has a witness $c$, and (ii) every constant $c$ is required by some $p$ in $A$.

$$
p=p \wedge 1=p \wedge\left(\bigvee_{i} t_{i}\right)=\bigvee_{i}\left(p \wedge t_{i}\right)
$$


Now we simply choose $x=\left(p_{1 j_{1}}, \cdots, p_{n j_{n}}\right)$ so that whenever $p \wedge t_{i} \neq 0$, then $p_{i j_{i}} \leqq p \wedge t_{i}$, and then $c_{x}$ is a witness for $p$. For then

$$
c_{x}(p)=\underset{\left\{i \mid p \wedge t_{i \neq 0\}}\right.}{\bigvee} t_{i},
$$

but this is just $\exists p$, by $(* *)$.

(ii) Given $c, x_{c}=\left(p_{1 j_{1}}, \cdots, p_{n j_{n}}\right)$, let $p=V_{i} p_{i j_{i}}$. Then $x_{c}$ satisfies the condition prescribed above in (i), so $c$ is a witness for $p$. Suppose $d$ is also a witness for $p$. $d\left(p_{i j}\right)$ is either 0 or $t_{i}$ as we saw in the discussion preceding this theorem. By $(* *) 1=\exists p$, so,

$$
1=\underset{\left\{i \mid d\left(p_{i j_{i}}\right)=t_{i}\right\}}{V} t_{i}
$$

and therefore no $t_{i}$ may be omitted in this supremum. Finally, then, $x_{d}=\left(p_{1 j_{1}}, \cdots, p_{n j_{n}}\right)=x_{c}$, so $d=c$.

Before proceeding to the main result I might remark that many of the results of this section extend with no essential change in technique to complete atomic algebras. Moreover, using a generalization of the above methods, where the notion of atom is replaced by that of maximal filter, one can prove that any monadic algebra $A$ which is a countable ascending union of monadic subalgebras, $A_{n}$, such that $\exists\left(A_{n}\right)$ is finite for all $n$, is rich. This class includes, in particular, all $A$ for which $\exists(A)$ is finite, and also, by Corollary 3 , all countable $A .^{3}$

3. Finitely generated free monadic algebras. Given any integer $r>0$, let $n=2^{r}, m=2^{(n-1)}$, and choose $n$ pairwise disjoint sets $S_{i}$, $i=1, \cdots, n$, each having $m$ elements. In each $S_{i}$ we may, by $(*)$, select $n-1$ subsets $S_{i j}, 1 \leqq j \leqq n, j \neq i$, which freely generate the Boolean algebra $B_{i}$ of all subsets of $S_{i}$. Now $\left\{S_{i} \mid i=1, \cdots, n\right\}$ is a partition (in the usual set-theoretic sense) of $S=\bigcup_{i=1}^{n} S_{i}$. Applying (*) again, let $R=\{1, \cdots, n\}$ and choose $r$ subsets, $R_{1}, \cdots, R_{r}$ of $R$ that freely generate the Boolean algebra of all subsets of $R$. Finally, we let $A$ be the Boolean algebra of all subsets of $S$, and we write

$$
p_{i}=\bigcup_{j \in R_{i}} S_{j} ; \quad i=1, \cdots, r .
$$

$A$ will be the main object of interest here, for our program is to introduce a quantifier on $A$ and then to prove that the $p_{i}$ just defined freely generate $A$. If $A_{0}$ is the Boolean subalgebra generated by $\left\{p_{1}, \cdots, p_{r}\right\}$, then clearly the atoms of $A_{0}$ are just $\left\{S_{1}, \cdots, S_{n}\right\}$. It is no loss to assume the $R_{i}$ indexed so that $\lambda_{P}\left(e^{k}\right)=S_{k}, k=1, \cdots, n$, where, of course, $P=\left(p_{1}, \cdots, p_{r}\right)$. That is to say,

\footnotetext{
${ }^{3}$ This observation is due to Professor Halmos.
} 


$$
\lambda P=\left(S_{1}, \cdots, S_{n}\right) .
$$

(3.2) We observe, before proceeding, that the algebra $A$ has evidently $n m$ atoms, (one element subsets of $S$ ).

Now write,

$$
\bar{S}_{i}=S_{i} \cup \underset{1 \leqq j \leqq n, j \neq i}{\cup} S_{j i}, \quad i=1, \cdots, n,
$$

and, using $(* *)$, define $\exists$ to be the quantifier induced by the (relatively complete) subalgebra $B$ generated by $\left\{\bar{S}_{1}, \cdots, \bar{S}_{n}\right\}$. We write $\bar{\lambda} \bar{P}=\left(\bar{S}_{1}, \cdots, \bar{S}_{n}\right)$ and note that $S_{i} \subset \bar{S}_{i}$ for each $i$. Let $A_{1}$ be the Boolean subalgebra generated by $\left\{S_{i}, \bar{S}_{i} \mid i=1, \cdots, n\right\}$. By Lemma 1 we have that

$$
\begin{aligned}
& \left\{S_{i} \cap \lambda_{\overline{\lambda P}}(e) \mid e \in E_{i}^{n}, \quad i=1, \cdots, n\right\} \text { is a partition of } 1 \\
& \text { containing the atoms of } A_{1} .
\end{aligned}
$$

Lemma 3. For all e in $E_{i}^{n}, S_{i} \cap \lambda_{\overline{\lambda P}}(e) \neq \phi, i=1, \cdots, n$.

Proof. First expand.

$$
S_{i} \cap \lambda_{\overline{\lambda P}}(e)=S_{i} \cap\left[\bar{S}_{1}^{e_{1}} \cap \cdots \cap \bar{S}_{n}^{e_{n}}\right]=\bigcap_{j=1}^{n}\left(S_{i} \cap \bar{S}_{j}^{e j}\right) .
$$

Now $\bar{S}_{j}=S_{j} \bigcup_{1 \leqq k \leqq n, k \neq j} S_{k j}$, and $S_{i} \cap S_{k j}$ is $S_{i j}$ or $\phi$ according as $k=\imath$ or $k \neq i$, (for $j \neq i$ ), so $S_{i} \cap \bar{S}_{j}=S_{i} \cap S_{i j}$, and, similarly, $S_{i} \cap \bar{S}_{j}^{-1}$ $=S_{i} \cap S_{i j}^{-1}$. For $e$ in $E_{i}^{n}, e_{i}$ is 1 , and $S_{i} \cap \bar{S}_{i}=S_{i}$, so in the above expansion,

$$
S_{i} \cap \lambda_{\overline{\lambda P}}(e)=S_{i} \cap \bigcap_{1 \leqq j \leqq n, j \neq i}\left(S_{i} \cap S_{i j}^{e_{j}}\right)=S_{i} \cap \bigcap_{1 \leqq j \leqq n, j \neq i} S_{i j}^{e_{j}}
$$

But this is just a typical atom (hence one element subset) of $B_{i}$.

By this lemma none of the $n m$ subsets in the collection $\left\{S_{i} \cap \lambda_{\overline{\lambda P}}(e) \mid e \in E_{i}^{n}, i=1, \cdots, n\right\}$ are empty, and, by (3.3), they are pairwise disjoint. Hence, by (3.2) and a simple application of the "pigeon-hole principle" the above collection must consist precisely of the set of all atoms of $A$.

Lemma 4. $A_{1}=A$.

Lemma 5. $\exists S_{k}=\bar{S}_{k}, k=1, \cdots, n$; i.e., $\bar{\lambda} \bar{P}=\exists \lambda P$.

Proof. By $(* *) \exists S_{k}$ is the supremum of the atoms of $B$ that $S_{k}$ intersects nontrivially. The atoms of $B$ are $\left\{\lambda_{\overline{\lambda P}}(e) \mid e \in E^{n}\right\}$. Lemma 3 shows that those atoms with $e$ in $E_{k}^{n}$ intersect $S_{k}$, and so $\bar{S}_{k}$ $=\bigcup_{e \in E_{k}^{n}} \lambda_{\overline{\lambda P}}(e) \subset \exists S_{k}$. However, $S_{k} \subset \bar{S}_{k} \in B$, so $\bar{S}_{k}$ is in $B\left(S_{K}\right)$, and 
hence $\exists S_{k}=\cap B\left(S_{k}\right) \subset \bar{S}_{k}$.

We now gather our results. By Theorem 1, Lemma 5, and Lemma 4, $\left\{p_{1}, \cdots, p_{r}\right\}$ is a monadic generating set for $A$. Moreover, by Lemma 3 and Lemma 5 ,

$$
\mu_{\lambda P, i}(e)=S_{i} \cap \lambda_{\overline{\lambda P}}(e) \neq \phi
$$

for any $e$ in $E_{i}^{n}, i=1, \cdots, n$. Therefore, the hypothesis of Theorem 2 is vacuously satisfied for any $\operatorname{map} f_{0}$ of $\left\{p_{1}, \cdots, p_{r}\right\}$ into some other monadic algebra. We have thus completed the construction.

Theorem 4. The monadic algebra $A$ above is free on the $r$ generators $\left\{p_{1}, \cdots, p_{r}\right\} . A$ has nm atoms, and so has

$$
2^{n m}=2^{\left[2^{r} 2^{\left(2^{r}-1\right)}\right]}
$$

elements.

We mention, without proof, a few of the properties of this algebra, $A$. First, $B=\exists(A)$ is a Boolean algebra with $2^{n}-1$ atoms (so is not free). Further, for each $k=1, \cdots, n$ there are $C_{n, k}$ atoms of $B$ which contain precisely $k$ atoms of $A$. Therefore, by Theorem $3, A$ has $N(A)=\prod_{k=1}^{n} k^{C_{n, k}}$ constants.

As a final remark, we might state the characterization of finite monadic algebras analogous to that given for Boolean algebras at the outset of this paper.

Theorem 5. If $A$ is a finite monadic algebra, then $A$ is determined up to isomorphism by the number $n$ and the additive partition, $n$ $=n_{1}+\cdots+n_{k}$, of $n$, where $n$ is the number of atoms of $A, k$ the number of atoms of $\exists(A)$, and, under some enumeration of the latter, the ith atom of $\exists(A)$ contains $n_{i}$ atoms of $A, i=1, \cdots, k$.

Proof. Let $S_{1}, \cdots, S_{k}$ be $k$ disjoint sets such that $S_{i}$ has $n_{i}$ elements, and let $B$ be the monadic algebra of all subsets of $S$ $=S_{1} \cup \ldots \cup S_{k}$, where the quantifier is that induced by the Boolean subalgebra generated by $\left\{S_{1}, \cdots, S_{k}\right\}$, (see $\left.(* *)\right)$. Now map the set $S$ (of all the $n$ atoms of $B$ ) one-to-one onto the atoms of $A$ in such a way that $S_{i}$ maps onto the atoms of $A$ below the $i$ th atom of $\exists(A)$. By Corollary 4, this map defines a Boolean homomorphism from $B$ to $A$ which, by (**) again is monadic. This monadic homomorphism is also evidently one-to-one and onto, so the proof is complete.

\section{REFERENCES} 1948.

1. G. Birkhoff, Lattice theory, Amer. Math. Soc. Colloquium Publications, vol. 25, 
2. G. Birkhoff and S. MacLane, A survey of modern algebra, MacMillan, 1953.

3. P. R. Halmos, Algebraic Logic, I-Monadic Boolean algebras, Compositia Math. vol. 12 (1955) pp. 217-249.

4. J. C. C. McKinsey and Alfred Tarski, The algebra of topology, Ann. of Math. vol. 45 (1944) pp. 141-191.

University of Chicago

\section{ON HARMONIC MAPPINGS ${ }^{1}$}

JOHANNES C. C. NITSCHE

1. Suppose that the functions $x=x(\alpha, \beta), y=y(\alpha, \beta)$ define a oneto-one harmonic mapping of the unit disc $\Gamma$ in the $\alpha, \beta$-plane $(\alpha+i \beta$ $=\gamma$ ) onto a convex domain $C$ in the $x, y$-plane $(x+i y=z)$. The origin is assumed to be fixed. Introducing two functions $F(\gamma)$ and $G(\gamma)$ which, in $\Gamma$, depend analytically upon the variable $\gamma$ we may write $z=\operatorname{Re} F(\gamma)+i \operatorname{Re} G(\gamma)$. The purpose of the present paper is (i) to give a new proof of a lemma which, in a special form, was first used by T. Radó [13] and which was proved in general by L. Bers (see [2, Lemma 3.3]), ${ }^{2}$ (ii) to derive an improved value for an important constant first introduced by $\mathrm{E}$. Heinz [3]. The proofs will be very simple due to the fact that there is a close connection between univalent harmonic mappings and the minimal surface equation (see e.g. $[11$, footnote 2]) and also the differential equation

$$
\phi_{x x} \phi_{y y}-\stackrel{2}{\phi_{x y}}=1 .
$$

The connection with the latter equation was exploited by K. Joergens [8] for the study of the solutions of (1). One can, however, proceed one step further by introducing a mapping which was invented by $\mathrm{H}$. Lewy $[10]$ for Monge-Ampère equations.

2. Let $z=\operatorname{Re} F(\gamma)+i \operatorname{Re} G(\gamma)$ be a harmonic mapping with the properties mentioned above. Then the expression

Presented to the Society August 20, 1956 and November 17, 1956; received by the editors September 10, 1957.

1 This paper was prepared under Contract $\mathrm{N}$ onr-710(16) between the University of Minnesota and the Office of Naval Research.

${ }^{2}$ It has been shown by H. Hopf (cf. [7, p. 133 and 5, pp. 91-92]) that the combination of Heinz's inequality with Schwarz's lemma yields a sharper result. 